\title{
Moisture Buffering and Mould Growth Characteristics of Naturally Ventilated Lime Plastered Houses.
}

\author{
Vismaya Paralkara ${ }^{a}$ Dr.Rashmin Damle ${ }^{\text {* }}$
}

a CEPT University, Ahmedabad, India

\begin{abstract}
Lime plaster is well known for its moisture buffering capabilities but is also susceptible to mould growth. This work focusses on the hygrothermal performance of lime plaster in naturally ventilated residential spaces. Surveys are carried out for 45 traditional buildings of Ahmedabad in India with measurements of ambient variables, such as temperature, relative humidity, wall moisture content, etc. Mould growth patterns of these spaces are related to the measured variables and wall characteristics. Hygrothermal simulations of some spaces are also carried out to observe the moisture buffering of lime plaster. Experimental observations are contrasted with simulation results to see if numerical predictions are realistic.
\end{abstract}

Peer-review under the responsibility of the organizing committee of the ICMB21.

Keywords: Lime plaster; Hygrothermal simulations; mould growth; surface relative humidity conditions.

\section{Introduction/Background}

Lime plaster is one of the key sustainable building materials that is also effective as a passive cooling strategy[1,2]. It has good vapour permeability that moderates the indoor relative humidity inside a space[3]. The moisture buffering quality of lime and its permeability results in the adsorption and desorption of space moisture [4]. It is highly influential in the moisture transfer of a building envelope. This results in a decrease in indoor relative humidity but at the same time, there is a risk of damaging the envelope. Lime plaster has a self-healing quality[5], durability and a longer life span. Hence, it is essentially used in conservation projects and vastly adds up to the qualities of the old building. As specified by [6], in old structures an important function is the perhaps vapour permeability of the ceiling and walls. The presence of organic mixes and moisture buffering quality of lime plaster makes it highly prone to phenomena like mould growth on the surface of the walls. Mould growth further degrades the indoor air quality and the occupant health is compromised. To avoid mould related problems, it is necessary to understand the behaviour of lime plaster with respect to the indoor relative humidity and surface moisture content.

A lot of information is available on the advantages of having lime plaster as a passive sustainable technique [7]. However, the literature lacks studies about the post-occupancy behaviour of lime plastered buildings in terms of their indoor environment and mould growth characteristics. This information can support conservation projects to maintain their longevity and improving their indoor air quality. This work attempts to evaluate the state of traditional lime plastered buildings in Ahmedabad and correlates the findings in terms of the building characteristics like coatings on the wall, ventilation, the level of water activity inside the space, function of the space, etc. Simulations are also carried out to understand the hygrothermal performance of lime plaster. Finally, experiments are also carried out to study the onset of mould growth in lime plaster samples.

\subsection{Methodology:}

The hygrothermal performance of lime plaster is observed through field measurements and simulations. Surveys of residential spaces are carried out in the afternoon from 02:00 pm to 04:00 pm when the temperatures are relatively high outside on every alternate day from the 25th of December 2019. Point in time measurements of the outdoor and indoor air temperature, relative humidity, globe temperature, moisture content of walls, and air velocity are noted for each space. The walls affected by mould are marked and the pattern of influencing factors is observed. The instruments used for measurements are Extech Heat Stress Meter, Peakmeter Vane Anemometer, FLIR Thermal Gun, and Testo 606-2 Moisture Meter. Hygrothermal performance is predicted by carrying out annual simulations with the Effective Moisture Penetration Depth (EMPD) model of Energy Plus [8] and contrasted with the simulation results observed on site. To understand the onset of mould growth lime plaster samples are exposed to salt solutions that maintain different relative humidity (RH) levels from $75 \%$ to $97 \%$.

\subsection{Observations:}

Figure 1a shows the results of the hygrothermal simulation of the BESTEST geometry [9] with different surface finishes. It is observed that lime plaster can effectively modulate the indoor relative humidity. Since all the parameters considered are the same except for the material properties, the influence of thermal mass on temperature and relative humidity modulation can be 


\section{ICMB21}

eliminated. Hygrothermal simulation of an onsite lime plastered space is shown in Figure 1b. The amplitude of outdoor relative humidity levels is higher as compared to the indoor levels. The reducing amplitude shows the moisture buffering due to lime plaster and is seen between maximum and minimum of daily relative humidity values. The red dotted box highlights the monsoon months when the relative humidity is high and favourable span for mould growth.
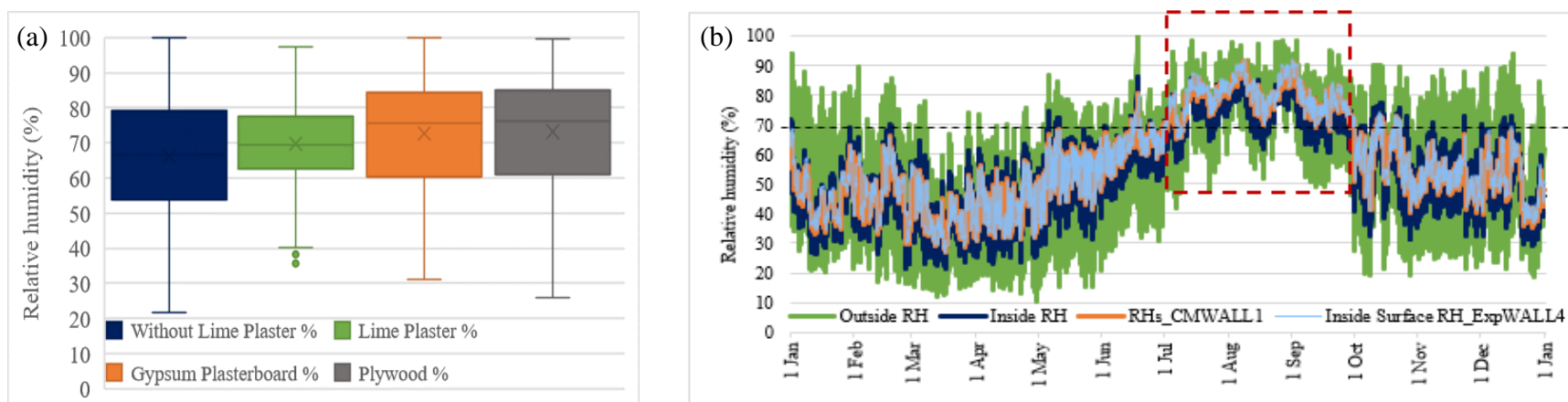

Figure 1 (a) Indoor Relative Humidity for different cases (b) Varying RH levels with time throughout the year in a simulated surveyed space

The above understanding is then applied to onsite observation of the mould affected walls. It helped to comprehend the factors that were highly influential on the mould growth. Onsite moisture content measurement indicates the mould being induced during monsoon and continued to breed due to trapped moisture in the walls because of various factors mentioned in Figure 2. It summarizes the strongest and common factors that affect mould growth based on field observations. The spaces highlighted in black have walls affected by mould. It is observed that due to lack of ventilation and sunlight the moisture trapped inside the space leads to mould growth. The best combination is where lime plaster is not coated or coated with lime-wash with well-ventilated and sunlit spaces. Mould growth inside lime plaster is observed if the moisture transfer is obstructed by any surface coating, object, or lack of ventilation. If lime plaster is allowed to breathe, favourable conditions for mould growth can be avoided. The salt solution experiments showed no visible mould growth till the third week for RH levels of $75 \%, 80 \%$, and $86 \%$. However, mould growth was visibly seen on the lime plaster sample after three weeks for an RH level of $96 \%$.

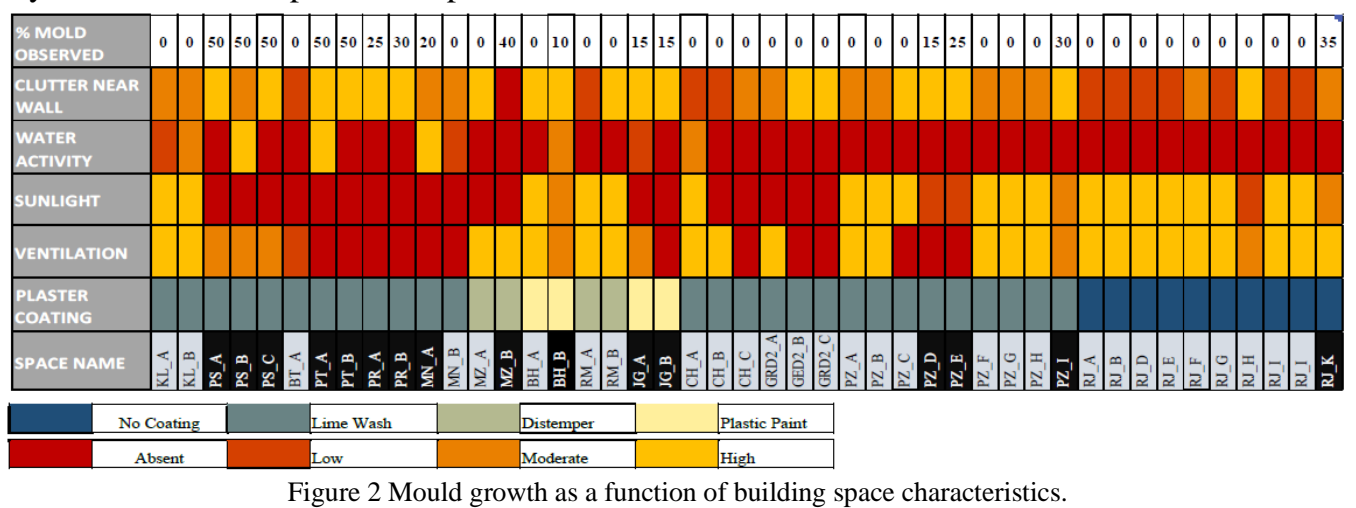

\subsection{Conclusion:}

The moisture buffering capacity of lime plaster is beneficial to regulate indoor relative humidity. However, one of the most important aspects is to prevent moisture from getting trapped in building fabric. With appropriate ventilation and allowing the lime plastered surface to breathe, lime plaster is one of the most sustainable building materials that can be used even today.

\section{References}

[1] [1] E. V. LYON, “The Literature of Cement, Lime, Plaster, and Gypsum,” pp. 122-141, 1968.

[2] [2] M. Doctor-Pingel, V. Vardhan, and et al., "Use of Lime Mortar and Post-Occupancy Thermal Performance Analysis of Buildings," Elsevier Inc, 2019.

[3] [3] L. Toniolo, A. Paradisi, S. Goidanich, and G. Pennati, "Mechanical behaviour of lime-based mortars after surface consolidation," Constr. Build. Mater., vol. 25 , no. 4 , pp. $1553-1559,2011$

[4] [4] P. Mold and R. Godbey, "Limewash : Compatible Coverings for," Group, pp. 1-11, 2005.

[5] [5] C. De Nardi, S. Bullo, A. Cecchi, and L. Ferrara, "Self-healing capacity of advanced lime mortars," Adv. Mater. Process. Technol., vol. 2, no. 3, pp. $349-360,2016$

[6] [6] R. Homes, B. Y. Roger, and H. June, "Expert guide to plaster in old homes," pp. 1-11, 2020.

[7] [7] K. Elert, C. Rodriguez-Navarro, Pardo, and et al., "Lime mortars for the conservation of historic buildings," Stud. Conserv., vol. 47, no. 1, pp. 62-75, 2002.

[8] [8] Energy Plus, “EnergyPlus 9.2.” 2019.

[9] [9] R. D. Judkoff and J. Neymark, "The BESTEST Method for Evaluating and Diagnosing Building Energy Software," ACEE Summer Study Energy Effic. Build., pp. 175-192, 1998. 